Special Issue: Diabetic Nephropathy

\title{
A Systematic Review of Metabolite Profiling in Diabetic Nephropathy
}

\author{
Xiaotian Liu ${ }^{1}$, Chao Liu ${ }^{1}$, Libin Zhan² and Lin Zhang1* \\ ${ }^{1}$ Academy of Integrative Medicine, Dalian Medical University, Dalian 116044, China \\ ${ }^{2}$ Department of Traditional Chinese Medicine, the Second Affiliated Hospital, Dalian Medical University, Dalian 116044, China
}

Received: March 03, 2015; Accepted: June 13, 2015; Published: June 25, 2015

*Corresponding author: Lin Zhang, Academy of Integrative Medicine, Dalian Medical University, Dalian 116044, China, Tel: +81 15942632527;

Email: zhl8247@163.com

\begin{abstract}
In recent years, the incidence of diabetic nephropathy (DN) is in sharply rising trend. It is a great challenge for health care professionals to prevent the DN.As the tool of modern bioinformatics research, metabonomics is of great significance for the early diagnosis of disease. In order to have a discussion about this, we conducted a systematic search of MEDLINE (PubMed) up using the key term combinations of 'metabolomics' and 'metabonomics' with 'diabetic nephropathy'. Finally we selected 18 articles. These researches help us to find more sensitive biomarkers to contribute to the diagnosis and therapeutic treatment of DN at an early phase and provide some ideas for the research of DN. This systematic review mainly summarized the current state of research on differential biomarkers of DN and discussed the clinical relevance of metabolomics in the prediction, diagnosis and management of DN.
\end{abstract}

Keywords: Metabolomics; Metabonomics; Diabetic Nephropathy; Biomarkers

\section{Introduction}

Diabetic Nephropathy (DN) refers to that various causes lead to the absolute or relative lack of insulin, resulting in glucose, protein and fat metabolism disorders, and it is a systemic disease based on main clinical manifestations of chronic high blood sugar. As one of the most common clinical diabetic complications, the incidence of DN is also on the rise in our country, and it has become the second cause of end-stage renal disease, just next to various glomerular nephritis [1]. It is also one of the major reasons for death and disability in patients with diabetes mellitus. Its mechanism is much complicated, and still not entirely clear, but it is generally believed that continuous renal hemodynamic changes caused by high blood sugar, sugar metabolic abnormalities associated with protein and fat metabolism disorder are the foundation of DN pathogenesis. Its clinical manifestations are related to the course of the disease, based on proteinuria as a prominent feature. Once the DN progressed to end-stage kidney disease, treatment is often much more difficult than other kidney disease. The current clinical treatment of DN includes reducing blood sugar and blood pressure, regulation of lipid metabolism disorders and low protein diet, etc. With the deeper development of science and technology, much more new theories and technologies applied to DN. Because of its characteristics with various metabolic disorders, metabolomics studies appear more prominent.

Metabonomics is a new field which develops after genomics and proteomics recently [2]. Genomics is to have a study of biological genome. Proteomics is to study all proteins of an organism or a tissue at the molecular level. And metabonomics is to have qualitative and quantitative analysis of low molecular weight metabolites in the cells or organisms at the same time. Disease often leads to the change of the body's physiological process, resulting in the corresponding changes in the metabolic products. We could analyze some metabolites in the urine, blood and other body fluids, and compare them with normal metabolic products to find biomarkers of disease and provide certain methods for the diagnosis and treatment [3]. But the studies of genes and proteins are unable to come to the conclusion like that. In addition, the main performance of the DN is the body's metabolic disturbance, therefore the metabonomic study of DN is very important. The methods like nuclear magnetic resonance (NMR), gas chromatography-mass spectrometry (GC/MS) and liquid chromatography-mass spectrometry (LC/MS) were often used as a tool in metabonomic study [4]. After that, it could provide certain methods and indicators for the early diagnosis of DN, the monitoring of development process, and the relief of the symptoms.

With the improvement of the people's living standard and changes in dietary structure, the numbers of the population with diabetes is increasing. A recent study predicted that by the year of 2030, a $42 \%$ increase in type 2 diabetes mellitus (T2DM) prevalence can be expected in developed countries, mainly among persons over 60 years of age [5]. So the prevention and treatment of DN is of great significance at international level. Determination of micro-albuminuria is the main method of clinical diagnosis of early DN currently. Some studies have even pointed out that detection of the albuminuria at different times have different value in the diagnosis of early renal damage in DN. For example, 
night albuminuria test is more accurate and more meaningful [6]. Serum creatinine and glomerular filtration function are also the indicators of renal damage in patients with diabetes, but albumin often can be found until the DN in clinical phase 3 when the treatment has been very difficult. Therefore looking for more sensitive and reliable clinical index for early diagnosis and treatment of DN has been the hot point in clinical study of DN. In recent years, research about DN patients' metabolites in serum, urine and other body fluids developed rapidly which opened up a new pathway in the research of DN. The purpose of this paper is to have a comprehensive summary on the relative metabolomics research of DN and provide some new ideas about the diagnosis and treatment of DN based on metabonomics in detail.

\section{Methods}

\section{Search strategy}

Literature searches of MEDLINE (PubMed) for relevant articles were conducted up with the key term combinations of 'metabolomics'or 'metabonomics' with 'Diabetic nephropathy'. To minimize selection bias, two investigators independently reviewed titles, abstracts and available full-text articles for relevance. Additional articles were identified through searching the reference lists from included studies. Disagreements were resolved by consensus and by a third investigator (Figure 1).

\section{Prespecified inclusion criteria}

(1) Article is original instead of review.

(2) Metabolomic techniques such as LC-MS or NMR spectroscopy were used to construct metabolite profiles.

(3) Evaluated compounds are metabolites in serum, urine, etc.

(4) Articles are in English.

\section{Data extraction and analysis}

Data on techniques and study results were extracted. One investigator performed the data extraction, which was verified by a second investigator.

\section{Results}

The selection algorithm for the 18 studies which met the inclusion/exclusion criteria for our systematic review is detailed in Fig. 1. These 18 articles include 11 clinical researches and 7 animal model researches with rats, mice and monkeys. Eight studies are based on NMR, three studies are on the basis of GCMS, four studies are based on LC-MS, only one study is on the basis of CE-MS, and two studies are based on both GC-MS and LC-MS.

\section{Metabolomic analytical technologies}

Nuclear Magnetic Resonance (NMR): NMR refers to the resonance transition phenomenon between the magnetic energy level of the substance's nucleus system in a magnetic field, while the corresponding frequency electromagnetic radiation has an effect on them. High resolution NMR technology was used to detect the metabolome in complete organ or tissue cell. And then the corresponding metabolites information can be obtained to study the NMR spectrum of these metabolisms which can

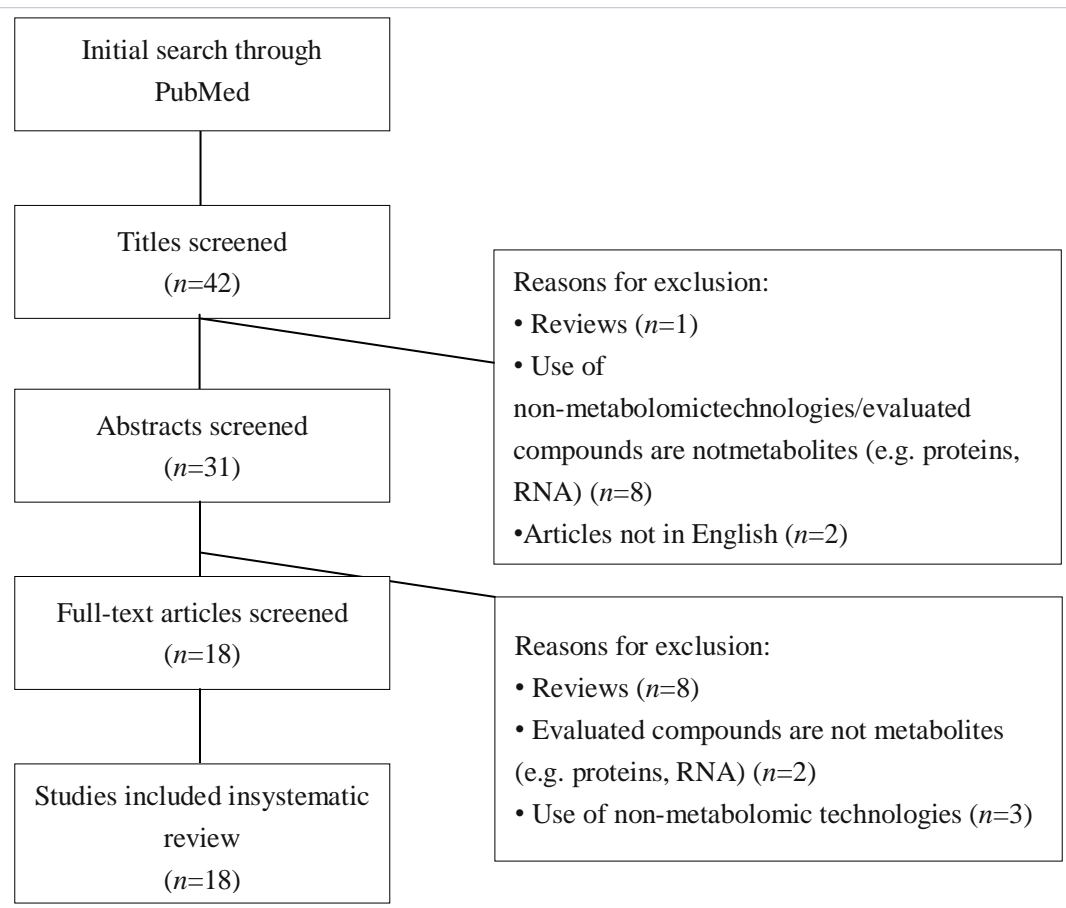

Figure 1: Systematic review study selection algorithm. The 18 studies that fulfilled the inclusion/ exclusion criteria for this systematic review were selected from among 42 articles identified in the initial PubMed literature review. The process for selecting the 18 articles is displayed. 
reflect some biological significance. It has the advantage of simple pretreatment method, short analysis time, high flux and no damage of the sample. But compared with mass spectrometry, the disadvantage is that the sensitivity and resolution is relatively low. Specific conditions of DN metabolomics are shown in Table 1.

Gas Chromatography-Mass Spectrometry (GC-MS): It was combined utilization of gas chromatography with mass spectrometry. Gas chromatography is the use of capillary column to separate the sample according to different chemical components. After the separated molecules across the mass spectrometer, its nature can be measured. That means gas chromatographic separates the mixtures, and mass spectrometry detects molecules. It has high sensitivity to detect a large number of small molecule metabolites with low concentration. It is suitable for the qualitative analysis of the unknown component in multicomponent mixture. Most of the samples need to have the derivatization under room temperature or heating conditions. Specific conditions of DN metabolomics are shown in Table 2.
Liquid Chromatography-Mass Spectrometry (LC-MS): LCMS is a technology which uses liquid chromatography to separate and mass spectrometry to detect. Based on the theory of classic chromatography, High-performance liquid chromatography (HPLC) changes the mobile phase into high pressure conveying in technology, separating on the chromatographic column, and detecting by the mass spectrometer. And compared with HPLC, Ultra high performance liquid chromatography (UPLC) has more flux, high sensitivity and more chromatographic peak capacity. Specific conditions of DN metabolomics are shown in Table 3.

Capillary Electrophoresis-Mass Spectrometry (CE-MS): Capillary electrophoresis is a kind of new technology. Capillary channel was used to separate, and high voltage direct current as a new driving force. Its biggest advantage was the little amount sample. The separation mechanism is different from the HPLC and GC. The ionic metabolites could be separated, and different metabolites map could also be obtained at the same time using CE. Specific conditions of DN metabolomics are shown in Table 4.

Table 1: Metabonomics condition of DN in NMR.

\begin{tabular}{|c|c|c|}
\hline Solutions & NMR & References \\
\hline $\begin{array}{l}400 \mu \mathrm{l} \text { urine mixed with } 100 \mu \mathrm{l} \text { PBS }(0.2 \mathrm{M}, \mathrm{pH}=7.0) \text { and } 50 \mu \mathrm{D}_{2} \mathrm{O} \text { containing TSP/ } 500 \mu \mathrm{l} \text { PBS with } \\
\text { addition of } 50 \mu \mathrm{l} \text { TSP }\left(1 \% \text {, in } \mathrm{D}_{2} 0\right) \text {,and } 550 \mu \mathrm{l} \text { renal supernatant }\end{array}$ & ${ }^{1} \mathrm{H}-\mathrm{NMR} / \mathrm{NOESY}$ & (Liu J et al.2015) \\
\hline $\begin{array}{l}400 \mu \mathrm{l} \text { urine mixed with } 100 \mu \mathrm{l} \text { phosphate buffer solution (PBS, } 0.2 \mathrm{M}, \mathrm{pH} 7.0 \text { ), with the addition of } \\
50 \mu \mathrm{l} 1 \%(\mathrm{w} / \mathrm{v}) \text { TSP dissolved in } \mathrm{D}_{2} \mathrm{O} \text {. }\end{array}$ & ${ }^{1} \mathrm{H}-\mathrm{NMR} / \mathrm{NOESY}$ & (Liu J et al.2013) \\
\hline $\begin{array}{l}300 \mu \mathrm{l} \text { of aliquots (Serum and urine) were mixed with } 300 \mu \mathrm{l} \text { of phosphate buffer/the renal tissue } \\
\text { extractswereresuspended in } 600 \mu \mathrm{l} \text { of phosphate buffer with } 60 \mu \mathrm{l} \text { of } \mathrm{D}_{2} \mathrm{O}\end{array}$ & ${ }^{1} \mathrm{H}-\mathrm{NMR}$ & (Zhao L et al.2012) \\
\hline $\begin{array}{l}100 \mu \mathrm{l} \text { of urine was mixed with } 41 \mu \mathrm{l} \text { of combination of } 70 \mathrm{mM} \text { sodium phosphate buffer, TSP, and } \\
\mathrm{NaN} 3 \text {, and } 59 \mu \mathrm{l} \text { of } 90 / 10 \% \mathrm{H}_{2} \mathrm{O} / \mathrm{D}_{2} \mathrm{O}\end{array}$ & $\begin{array}{l}{ }^{1} \mathrm{H}-\mathrm{NMR} / 2 \mathrm{D}-\mathrm{NMR} / \\
{ }^{13} \mathrm{C} \text { NMR/COSY }\end{array}$ & (Stec DF et al.2015) \\
\hline $\begin{array}{l}430 \mu \mathrm{L} \text { of each serum samples was mixed with the reference substance (TSP } 40 \mathrm{mmol} / \mathrm{l}, \mathrm{MnSO}_{4} 0.6 \\
\mathrm{mmol} / \mathrm{l} \text { in } 99.8 \% \mathrm{D}_{2} \mathrm{O} \text { ) }\end{array}$ & ${ }^{1} \mathrm{H}-\mathrm{NMR}$ & $\begin{array}{l}\text { (Makinen V et } \\
\text { al.2008) }\end{array}$ \\
\hline $\begin{array}{l}430 \mu \mathrm{L} \text { of each serum samples was mixed with the reference substance (TSP } 40 \mathrm{mmol} / \mathrm{l}, \mathrm{MnSO}_{4} 0.6 \\
\mathrm{mmol} / \mathrm{l} \text { in } 99.8 \% \mathrm{D}_{2} \mathrm{O} \text { ) }\end{array}$ & ${ }^{1} \mathrm{H}-\mathrm{NMR}$ & $\begin{array}{l}\text { (Mäkinen V et } \\
\text { al.2006) }\end{array}$ \\
\hline $\begin{array}{l}430 \mu \mathrm{L} \text { of each serum samples was mixed with the reference substance (TSP } 40 \mathrm{mmol} / \mathrm{l}, \mathrm{MnSO}_{4} 0.6 \\
\mathrm{mmol} / \mathrm{l} \text { in } 99.8 \% \mathrm{D}_{2} \mathrm{O} \text { ) }\end{array}$ & ${ }^{1} \mathrm{H}-\mathrm{NMR}$ & $\begin{array}{l}\text { (Makinen V et } \\
\text { al.2012) }\end{array}$ \\
\hline
\end{tabular}

Table 2: Metabonomics condition of DN in GC-MS.

Chromatographic column

$10 \mathrm{~m} \times 0.18 \mathrm{~mm}$ inner diamteter fused-silica capillary column chemically bonded with $0.18 \mu \mathrm{m}$ DB5-MS stationary phase (J\&W Scientific, Folsom, CA)

DB-5ms capillary column $(30 \mathrm{~m} \times 250 \mu$ mid., 0.25 $\mu \mathrm{m}$ film thickness; Agilent J\&W Scientific, Folsom, CA, USA)

VF-5ms capillary column $(30 \mathrm{~m} \times 0.25 \mathrm{~mm}$ id.; film thickness $0.25 \mu \mathrm{m}$ )

$5 \%$ phenyl dimethyl silicone

$30 \mathrm{~m} \times 0.32 \mathrm{~mm}$ column(Agilent DB-5)

\begin{tabular}{|c|c|c|c|c|c|c|}
\hline $\begin{array}{c}\text { Injection } \\
\text { temperature }\end{array}$ & $\begin{array}{c}\text { Initial } \\
\text { column } \\
\text { temperature }\end{array}$ & Flow rate & $\begin{array}{c}\text { Ion source } \\
\text { temperature }\end{array}$ & $\begin{array}{c}\text { Electron } \\
\text { energy }\end{array}$ & Scan range & References \\
\hline $250^{\circ} \mathrm{C}$ & $70^{\circ} \mathrm{C}$ & $\begin{array}{c}1.0 \\
\mathrm{ml} / \mathrm{min}\end{array}$ & $200^{\circ} \mathrm{C}$ & $70-\mathrm{eV}$ & $50-680 \mathrm{~m} / \mathrm{z}$ & $\begin{array}{c}\text { (Li M et al. } \\
2013)\end{array}$ \\
\hline $270^{\circ} \mathrm{C}$ & $80^{\circ} \mathrm{C}$ & $1.0 \mathrm{ml} / \mathrm{min}$ & $200^{\circ} \mathrm{C}$, & $70 \mathrm{eV}$ & $30-600 \mathrm{~m} / \mathrm{z}$ & $\begin{array}{c}\text { (Zhao T et al. } \\
2015)\end{array}$ \\
\hline $280^{\circ} \mathrm{C}$ & $90^{\circ} \mathrm{C}$ & $\begin{array}{c}1.0 \\
\mathrm{ml} / \mathrm{min}\end{array}$ & $200^{\circ} \mathrm{C}$ & - & $50-400 \mathrm{~m} / \mathrm{z}$ & $\begin{array}{c}\text { (Han L et al. } \\
2011)\end{array}$ \\
\hline- & $60^{\circ} \mathrm{C}$ & - & - & - & & $\begin{array}{c}\text { (Niewczas } \\
\text { MA et al. } \\
2014)\end{array}$ \\
\hline- & $70^{\circ} \mathrm{C}$ & - & - & - & & $\begin{array}{c}\text { (Sharma K et } \\
\text { al. 2013) }\end{array}$ \\
\hline
\end{tabular}


Table 3: Metabonomics condition of DN in LC-MS.

\begin{tabular}{|c|c|c|c|c|c|c|c|c|c|}
\hline LC-MS & Mobile phase A & Mobile phase B & $\begin{array}{l}\text { tem- } \\
\text { pera } \\
\text { ture }\end{array}$ & $\begin{array}{l}\text { chromatographic } \\
\text { column }\end{array}$ & $\begin{array}{l}\text { Injection } \\
\text { Volume }\end{array}$ & $\begin{array}{l}\text { flow } \\
\text { rate(ml/ } \\
\text { min) }\end{array}$ & voltage & $\begin{array}{l}\operatorname{scan} \\
\operatorname{range}(m / z)\end{array}$ & references \\
\hline $\begin{array}{l}\text { HPLC- } \\
\text { MS }\end{array}$ & - & - & $85^{\circ} \mathrm{C}$ & $\begin{array}{l}\text { SUPELCOGEL Pb } \\
(300 \times 7.8 \mathrm{~mm} ; 5 \mu \mathrm{m} ; \\
\text { Supelco UK) column }\end{array}$ & - & - & - & - & $\begin{array}{c}\text { (Niewczas } \\
\text { MA et } \\
\text { al.2014) }\end{array}$ \\
\hline $\begin{array}{l}\text { HPLC- } \\
\text { MS }\end{array}$ & $\begin{array}{l}10 \mathrm{mMammonium} \\
\text { acetate in Ultra } \\
\text { pure water } \\
\text { adjusted to pH } 5.8 \\
\text { with glacial acetic } \\
\text { acid }\end{array}$ & $100 \%$ methanol & $25^{\circ} \mathrm{C}$ & $\begin{array}{l}\text { Agilent TC-C18 column } \\
(250 \mathrm{~mm} \times 4.6 \mathrm{~mm} \\
5 \mu \mathrm{m} \text { size, Agilent } \\
\text { Technologies }) \text { with an } \\
\text { Alltech guard column } \\
(7.5 \mathrm{~mm} \times 4.6 \mathrm{~mm} \text { I.D., } 5 \mu \mathrm{m} \\
\text { particle size })\end{array}$ & $20 \mu \mathrm{L}$. & 0.8 & $\begin{array}{c}\text { ion spray } \\
\text { voltage:5KV. }\end{array}$ & - & $\begin{array}{l}\text { (Xia J et } \\
\text { al.2009) }\end{array}$ \\
\hline UPLC-MS & $0.1 \%$ formic acid & $\begin{array}{c}\text { acetonitrile } \\
\text { modified by the } \\
\text { addition } \\
\text { of } 0.1 \% \text { formic acid }\end{array}$ & $35^{\circ} \mathrm{C}$ & $\begin{array}{l}\text { 100mm×2.1mm i.d. } \\
\text { ACQUITY } \\
1.7 \mu \mathrm{mC} 18 \text { column }\end{array}$ & $5 \mu \mathrm{l}$ & 0.4 & $\begin{array}{l}\text { capillary } \\
\text { voltage: } 2.4 \mathrm{kV} \\
\text { conevoltage: } \\
35 \mathrm{~V} \\
\end{array}$ & 100 to 1000 & $\begin{array}{l}\text { (Zhang J et } \\
\text { al.2009) }\end{array}$ \\
\hline UPLC-MS & $\begin{array}{l}\text { water with } 0.1 \% \\
\text { ammonium }\end{array}$ & acetonitrile & - & $\begin{array}{l}10 \mathrm{~cm} \times 2.1 \mathrm{~mm}, 1.7 \mu \mathrm{m} \\
\text { BEH C18 column } \\
(\text { Waters, USA) }\end{array}$ & $\begin{array}{l}2 \mu \mathrm{l} \text { for } \\
\mathrm{ES}^{+} \text {and } \\
4 \mu \mathrm{l} \text { for } \\
\mathrm{ES}^{-}\end{array}$ & 0.4 & $\begin{array}{l}\text { capillary } \\
\text { voltage: } 2.3 \mathrm{kV} \\
\text { conevoltage: } \\
40 \mathrm{~V}\end{array}$ & 50 to 600 & $\begin{array}{c}\text { (Zhao T et al. } \\
\text { 2015) }\end{array}$ \\
\hline UPLC-MS & $0.1 \%$ Formic acid & $\begin{array}{l}\text { acetonitrile with } \\
0.1 \% \text { formic acid }\end{array}$ & $30^{\circ} \mathrm{C}$ & $\begin{array}{l}\text { Kinetex, } \text { C18 column } \\
\text { Luna C-18 column }\end{array}$ & - & - & - & - & $\begin{array}{l}\text { (Niewczas } \\
\text { MA et al. } \\
2014)\end{array}$ \\
\hline
\end{tabular}

\section{Data analysis for metabolomics}

Metabolomics data of DN is complex and diverse, the appropriate data analysis tools should be used to screen more meaningful data and get more useful information, meanwhile the metabolic processes and the related mechanism could be described better. Recently, the common methods of data analysis are PCA, PLS-DA and OPLS-DA, etc. The PCA is often used to observe the distribution of all data including the clustering and the outlier, and distinguish DN groups from control group clearly [7-16]. The PLS-DA is performed to visualize the systemically metabolic alterations among different groups and identify the response variables contributing most to the model and further identify potential markers of DN [10,12,14,17-19]. The OPLS-DA could be used to perform pattern recognition, and identify the changes of metabolites and potential biomarkers through the model analysis and nonparametric test $[7,8,20,21]$.

\section{Metabolomics for DN}

Metabolomics in clinical research: The samples from metabolomic study of DN often concentrated in the urine, serum or plasma in clinical. Details of metabolites are shown in Table 5. Some studies have found the significant correlations between the metabolites and the changes in urinary albumin excretion [22] or mitochondrial function [9]. These metabolites may be used as potential biomarkers for prediction of progression in DN. CE-MS-based metabolome analysis was also used to distinguish the severity of DN from non-DN, micro-DN, and macro-DN serum samples [20]. Taken together, a large number of clinical researches prove that the concentration of some differential metabolites and biomarkers have strong link with the development of DN.

It is important to note that the extent of changes in differential metabolites and biomarkers also played an important role in the studies. Investigators found that symmetric dimethylarginine (SDMA) in DN patient is a more sensitive marker than asymmetric dimethylarginine (ADMA), because ADMA was under the detection limit, but SDMA was positively correlated with a decrease in function of kidney [20]. And some researches indicated that the NEFAs' concentration shows little changes and the EFAs' levels increase from the stage of DM to the stage of DNIII [18].

In addition, several metabolic pathways were identified from metabolomics studies. Metabolites from the Krebs cycle, pyrimidine metabolism, amino acid, propionate, fatty acid, and oxalate metabolism were all significantly reduced in the urine of patients with diabetic kidney disease [9]. Investigators found significant accumulation of urea cycle intermediates in the patients with end-stage renal disease [20]. Glucosylceramide formation represented an important pathway for glucose metabolism in diabetic nephropathy [13]. And the purine and pyrimidine metabolic pathway is strongly associated with the development of DN [23].

Metabolomics in animal model research: The studies of urine, serum, plasma and tissue metabolites of DN were also 
Table 4: Metabonomics condition of DN in CE-MS.

\begin{tabular}{|c|c|c|c|c|c|c|c|c|c|}
\hline Ion & reference electrolyte & $\begin{array}{l}\text { capillary } \\
\text { voltage }\end{array}$ & temperature & $\begin{array}{l}\text { Sheath } \\
\text { liquid }\end{array}$ & $\begin{array}{l}\text { fragmenter } \\
\text { voltage }\end{array}$ & $\begin{array}{l}\text { skimmer } \\
\text { voltage }\end{array}$ & $\begin{array}{l}\text { octapole } \\
\text { radio } \\
\text { frequency } \\
\text { voltage }\end{array}$ & $\begin{array}{l}\operatorname{scan} \\
\operatorname{range}(m / z)\end{array}$ & references \\
\hline Cationic & $1 \mathrm{~mol} / \mathrm{L}$ formic acid & $4 \mathrm{KV}$ & \multirow[b]{2}{*}{$\begin{array}{c}\text { Capillary: } \\
20^{\circ} \mathrm{C} \text {; } \\
\text { sample trays : } \\
<5^{\circ} \mathrm{C}\end{array}$} & \multirow{2}{*}{$\begin{array}{c}\text { Methanol/ } \\
\text { water } \\
(50 \% \mathrm{v} / \mathrm{v}) \\
\text { containing } \\
0.1 \mu \mathrm{mol} / \\
\text { LHexakis }\end{array}$} & $70 \mathrm{~V}$ & $50 \mathrm{~V}$ & $125 \mathrm{~V}$ & \multirow[b]{2}{*}{$\begin{array}{l}50 \\
\text { to } 1000\end{array}$} & \multirow[b]{2}{*}{$\begin{array}{c}\text { (Hirayama A } \\
\text { et al.2012) }\end{array}$} \\
\hline Anionic & $\begin{array}{c}\text { Ammonium acetate } \\
\text { solution (50 mmol/L, } \\
\text { pH 8.5) }\end{array}$ & $3.5 \mathrm{KV}$ & & & $100 \mathrm{~V}$ & $50 \mathrm{~V}$ & $200 \mathrm{~V}$ & & \\
\hline
\end{tabular}

Table 5: Biomarkers reported in the context of DN in clinical research.

\begin{tabular}{|c|c|c|c|c|c|c|c|c|c|}
\hline Name & Source & Method & References & Tendency & Name & Source & Method & References & Tendency \\
\hline (Glycine, lysine) $\mathrm{UN}^{\mathrm{a}}$ & S & LC-MS & (Zhang ) & $\uparrow$ & MID 134 & S & CE-MS & (Hirayama) & $\downarrow$ \\
\hline $\begin{array}{l}\text { 2-ethyl 3-OH } \\
\text { propionate }\end{array}$ & $\mathrm{U}$ & GC-MS & (Sharma) & $\downarrow$ & MID 152 & S & CE-MS & (Hirayama) & $\uparrow$ \\
\hline 2-methyl acetoacetate & $\mathrm{U}$ & GC-MS & (Sharma) & $\downarrow$ & MID 158 & S & CE-MS & (Hirayama) & $\uparrow$ \\
\hline 3-hydroxy isobutyrate & $\mathrm{U}$ & GC-MS & (Sharma) & $\downarrow$ & MID 17 & S & CE-MS & (Hirayama) & $\uparrow$ \\
\hline 3-hydroxy isovalerate & $\mathrm{U}$ & GC-MS & (Sharma) & $\downarrow$ & MID 202 & S & CE-MS & (Hirayama) & $\uparrow$ \\
\hline 3-hydroxy propionate & $\mathrm{U}$ & GC-MS & (Sharma) & $\downarrow$ & MID 51 & S & CE-MS & (Hirayama) & $\downarrow$ \\
\hline 3-indoxyl sulfate & $\mathrm{P}$ & LC-MS & (Niewczas) & $\uparrow$ & Threitol & $\mathrm{P}$ & LC-MS & (Niewczas) & $\uparrow$ \\
\hline 3-methyl adipic acid & $\mathrm{U}$ & GC-MS & (Sharma) & $\downarrow$ & MID 52 & S & CE-MS & (Hirayama) & $\downarrow$ \\
\hline $\begin{array}{l}\text { 3-methyl crotonyl } \\
\text { glycine }\end{array}$ & $\mathrm{U}$ & GC-MS & (Sharma) & $\downarrow$ & MID 96 & S & CE-MS & (Hirayama) & $\downarrow$ \\
\hline Aconitic acid & $\mathrm{U}$ & GC-MS & (Sharma) & $\downarrow$ & MID 97 & S & CE-MS & (Hirayama) & $\uparrow$ \\
\hline Adenosine & $\mathrm{P}$ & LC-MS & (Xia) & $\uparrow$ & NEFA(C10:0) & $\mathrm{P}$ & GC-MS & (Han) & $\downarrow$ \\
\hline Apolipoprotein B-100 & S & NMR & (Mäkinen, 2006) & $\uparrow$ & NEFA(C12:0) & $\mathrm{P}$ & GC-MS & (Han) & $\downarrow$ \\
\hline Arabitol & $\mathrm{P}$ & LC-MS & (Niewczas) & $\uparrow$ & $\begin{array}{l}\text { N6-carbamoylthreo- } \\
\text { nyladenosine }\end{array}$ & $\mathrm{P}$ & LC-MS & (Niewczas) & $\uparrow$ \\
\hline Aspartic acid & S & CE-MS & (Hirayama) & $\uparrow$ & NEFA(C14:0) & $\mathrm{P}$ & GC-MS & (Han) & $\downarrow$ \\
\hline Azelaic acid & S & CE-MS & (Hirayama) & $\downarrow$ & NEFA(C16:0) & $\mathrm{P}$ & GC-MS & (Han) & $\downarrow$ \\
\hline butenoylcarnitine & $\mathrm{P}$ & LC-MS & (Pena) & $\uparrow$ & NEFA(C16:1n-9) & $\mathrm{P}$ & GC-MS & (Han) & $\downarrow$ \\
\hline Cholesterol & S & NMR & (Mäkinen, 2006) & $\uparrow$ & NEFA(C18:0) & $\mathrm{P}$ & GC-MS & (Han) & $\uparrow$ \\
\hline Citric acid & $\mathrm{U}$ & GC-MS & (Sharma) & $\downarrow$ & NEFA(C18:1n-11) & $\mathrm{P}$ & GC-MS & (Han) & $\uparrow$ \\
\hline Citrulline & S & CE-MS & (Hirayama) & $\uparrow$ & NEFA(C18:1n-9) & $\mathrm{P}$ & GC-MS & (Han) & $\downarrow$ \\
\hline C-peptide & $S$ & NMR & (Mäkinen, 2006) & $\uparrow$ & NEFA(C18:2) & $\mathrm{P}$ & GC-MS & (Han) & $\downarrow$ \\
\hline Cystatin-C & S & NMR & (Mäkinen, 2012) & $\uparrow$ & NEFA(C20:3) & $\mathrm{P}$ & GC-MS & (Han) & $\downarrow$ \\
\hline Cytidine & $\mathrm{P}$ & LC-MS & (Xia) & $\uparrow$ & NEFA(C20:4) & $\mathrm{P}$ & GC-MS & (Han) & $\uparrow$ \\
\hline Cytosine & $\mathrm{P}$ & LC-MS & (Xia) & $\uparrow$ & NEFA(C20:5) & $\mathrm{P}$ & GC-MS & (Han) & $\downarrow$ \\
\hline Deoxyuridine & $\mathrm{P}$ & LC-MS & (Xia) & $\uparrow$ & NEFA(C22:6) & $\mathrm{P}$ & GC-MS & (Han) & $\uparrow$ \\
\hline Dihydrosphingosine & S & LC-MS & (Zhang) & $\downarrow$ & Nitrogen & $\mathrm{U}$ & NMR & $\begin{array}{l}\text { (Mäkinen, } \\
\text { 2006) }\end{array}$ & $\downarrow$ \\
\hline Dimethylglycine & $\mathrm{P}$ & LC-MS & (Niewczas) & $\uparrow$ & $\begin{array}{l}\text { N2,N2- } \\
\text { dimethylguanosine }\end{array}$ & $\mathrm{P}$ & LC-MS & (Niewczas) & $\uparrow$ \\
\hline EFA(C10:0) & $\mathrm{P}$ & GC-MS & (Han) & $\downarrow$ & Orotic acid & $\mathrm{P}$ & LC-MS & (Xia) & $\uparrow$ \\
\hline
\end{tabular}




\begin{tabular}{|c|c|c|c|c|c|c|c|c|c|}
\hline $\mathrm{EFA}(\mathrm{C} 12: 0)$ & $\mathrm{P}$ & GC-MS & (Han) & $\uparrow$ & $\mathrm{PC}(\mathrm{C} 16: 0 / 18: 0)$ & $\mathrm{P}$ & LC-MS & (Zhu) & $\downarrow$ \\
\hline $\mathrm{EFA}(\mathrm{C} 14: 0)$ & $\mathrm{P}$ & GC-MS & (Han) & $\downarrow$ & $\mathrm{PC}(\mathrm{C} 16: 0 / 18: 2)$ & $\mathrm{P}$ & LC-MS & (Zhu) & $\uparrow$ \\
\hline EFA(C16:0) & $\mathrm{P}$ & GC-MS & (Han) & $\downarrow$ & $\mathrm{PC}(\mathrm{C} 18: 0 / 20: 4)$ & $\mathrm{P}$ & LC-MS & (Zhu) & $\downarrow$ \\
\hline EFA(C16:1n-9) & $\mathrm{P}$ & GC-MS & (Han) & $\downarrow$ & P-cresol sulfate & $\mathrm{P}$ & LC-MS & (Niewczas) & $\uparrow$ \\
\hline EFA(C18:0) & $\mathrm{P}$ & GC-MS & (Han) & $\downarrow$ & $\operatorname{PE}(\mathrm{C} 16: 0 / 18: 1)$ & $\mathrm{P}$ & LC-MS & $(\mathrm{Zhu})$ & $\uparrow$ \\
\hline EFA(C18:1n-11) & $\mathrm{P}$ & GC-MS & (Han) & $\uparrow$ & PE(C16:0/20:4) & $\mathrm{P}$ & LC-MS & (Zhu) & $\uparrow$ \\
\hline EFA(C18:1n-9) & $\mathrm{P}$ & GC-MS & (Han) & $\uparrow$ & PE(pC18:0/20:4) & $\mathrm{P}$ & LC-MS & (Zhu) & $\downarrow$ \\
\hline EFA(C18:2) & $\mathrm{P}$ & GC-MS & (Han) & $\downarrow$ & PG(C18:0/18:2) & $\mathrm{P}$ & LC-MS & (Zhu) & $\uparrow$ \\
\hline EFA(C20:0) & $\mathrm{P}$ & GC-MS & (Han) & $\uparrow$ & Phenylacetylglutamine & $\mathrm{P}$ & LC-MS & (Niewczas) & $\uparrow$ \\
\hline EFA(C20:2) & $\mathrm{P}$ & GC-MS & (Han) & $\uparrow$ & Phytosphingosine & S & LC-MS & (Zhang) & $\downarrow$ \\
\hline EFA(C20:3) & $\mathrm{P}$ & GC-MS & (Han) & $\downarrow$ & PI(C16:0/18:0) & $\mathrm{P}$ & LC-MS & (Zhu) & $\downarrow$ \\
\hline EFA(C20:4) & $\mathrm{P}$ & GC-MS & (Han) & $\uparrow$ & PI(C18:0/20:4) & $\mathrm{P}$ & LC-MS & (Zhu) & $\downarrow$ \\
\hline EFA(C20:5) & $\mathrm{P}$ & GC-MS & (Han) & $\downarrow$ & $\mathrm{PI}(\mathrm{C} 18: 0 / 22: 6)$ & $\mathrm{P}$ & LC-MS & (Zhu) & $\downarrow$ \\
\hline EFA(C22:6) & $\mathrm{P}$ & GC-MS & (Han) & $\uparrow$ & PS(C18:0/18:0) & $\mathrm{P}$ & LC-MS & (Zhu) & $\downarrow$ \\
\hline Erythritol & $\mathrm{P}$ & LC-MS & (Niewczas) & $\uparrow$ & N1-methyladenosine & $\mathrm{P}$ & LC-MS & (Niewczas) & $\uparrow$ \\
\hline Galactaric acid & S & CE-MS & (Hirayama) & $\downarrow$ & Pseudouridine & $\mathrm{P}$ & LC-MS & (Niewczas) & $\uparrow$ \\
\hline Glutamine & $\mathrm{U}$ & LC-MS & (Pena) & $\downarrow$ & SDMA & S & CE-MS & (Hirayama) & $\uparrow$ \\
\hline Glycolic acid & $\mathrm{U}$ & GC-MS & (Sharma) & $\downarrow$ & $\operatorname{SM}(\mathrm{dC} 18: 0 / 20: 2)$ & $\mathrm{P}$ & LC-MS & (Zhu) & $\uparrow$ \\
\hline HDL cholesterol & S & NMR & $\begin{array}{l}\text { (Mäkinen, 2008), } \\
\text { (Mäkinen, 2006) }\end{array}$ & $\downarrow$ & $\operatorname{SM}(\mathrm{dC} 18: 1 / 16: 0)$ & $P$ & LC-MS & (Zhu) & $\uparrow$ \\
\hline HDL2 cholesterol & S & NMR & (Mäkinen, 2006) & $\downarrow$ & Thymidine & $\mathrm{P}$ & LC-MS & (Xia) & $\uparrow$ \\
\hline HDL3 cholesterol & S & NMR & (Mäkinen, 2006) & $\downarrow$ & Thymine & $\mathrm{P}$ & LC-MS & (Xia) & $\uparrow$ \\
\hline Hexose & U & LC-MS & (Pena) & $\downarrow$ & Tiglylglycine & U & GC-MS & (Sharma) & $\downarrow$ \\
\hline Histidine & $\mathrm{P}$ & LC-MS & (Pena) & $\downarrow$ & Triglycerides & S & NMR & $\begin{array}{l}\text { (Mäkinen, } \\
\text { 2008/2006), }\end{array}$ & $\uparrow$ \\
\hline Homovanillic acid & $\mathrm{U}$ & GC-MS & (Sharma) & $\downarrow$ & Tyrosine & $\mathrm{U}$ & LC-MS & (Pena) & $\downarrow$ \\
\hline Hypoxanthine & $\mathrm{P}$ & LC-MS & (Xia) & $\uparrow$ & UN (C18H36NO) & S & LC-MS & (Zhang ) & $\uparrow$ \\
\hline Indoleacetate & $\mathrm{P}$ & LC-MS & (Niewczas) & $\uparrow$ & UN (C18H37N2O) & S & LC-MS & (Zhang ) & $\downarrow$ \\
\hline Inosine & $\mathrm{P}$ & LC-MS & (Xia) & $\uparrow$ & Urate & $\mathrm{P}$ & LC-MS & (Niewczas) & $\uparrow$ \\
\hline Kynurenate & $\mathrm{P}$ & LC-MS & (Niewczas) & $\uparrow$ & UN (C20H46N7O7) & $S$ & LC-MS & (Zhang ) & $\uparrow$ \\
\hline Kynurenine & S & CE-MS & (Hirayama) & $\uparrow$ & UN( C23H31N2O5) & S & LC-MS & (Zhang ) & $\downarrow$ \\
\hline Leucine & S & LC-MS & (Zhang) & $\downarrow$ & Uracil & U & GC-MS & (Sharma) & $\downarrow$ \\
\hline LPC (C16:0) & $\mathrm{P}$ & LC-MS & (Zhu) & $\uparrow$ & Urate & $\mathrm{P}$ & LC-MS & (Niewczas) & $\uparrow$ \\
\hline LPC (C18:0) & $\mathrm{P}$ & LC-MS & (Zhu) & $\uparrow$ & Uric acid & $P$ & LC-MS & (Xia) & $\uparrow$ \\
\hline LPC(C18:1) & $\mathrm{P}$ & LC-MS & (Zhu) & $\uparrow$ & Uridine & $\mathrm{P}$ & LC-MS & (Xia) & $\downarrow$ \\
\hline LPC(C18:2) & $\mathrm{P}$ & LC-MS & (Zhu) & $\uparrow$ & Waist-to-hip ratio & $S$ & NMR & $\begin{array}{l}\text { (Mäkinen, } \\
2006)\end{array}$ & $\uparrow$ \\
\hline LPC(C20:4) & $\mathrm{P}$ & LC-MS & (Zhu) & $\uparrow$ & Xanthine & $\mathrm{P}$ & LC-MS & (Xia) & $\uparrow$ \\
\hline MID 114 & S & CE-MS & (Hirayama) & $\downarrow$ & $\gamma$-butyrobetaine & S & CE-MS & (Hirayama) & $\uparrow$ \\
\hline MID 127 & S & CE-MS & (Hirayama) & $\downarrow$ & N4-acetylcytidine & $\mathrm{P}$ & LC-MS & (Niewczas) & $\uparrow$ \\
\hline $\begin{array}{l}\text { N1-Methyl-2-pyri- } \\
\text { done-5-Carboxamide }\end{array}$ & $\mathrm{P}$ & LC-MS & (Niewczas) & $\uparrow$ & & & & & \\
\hline
\end{tabular}

P: plasma; S: serum; U: urine; R: renal extracts.

a: Unknown.

included in animal model research (rat, mice and monkey). Details of metabolites are shown in Table 6. UA and allantoin may be used as potential markers for oxidative stress in DN by the research in mice [7]. Integrated information from serum and urine could reveal the systemic metabolic changes during the development of DN in a monkey model using ${ }^{1} \mathrm{H}$ NMR-based metabonomics [8]. In the rat model of DN, intrarenal metabolomics based on integrated GC-TOF MS and UPLC-TOF MS analytical platform was applied to find increasing intrarenal organic toxin including uremic toxins, glucuronides and glucotoxicity strongly associated with the progression of DN [17].
It is important to note that the extent of changes in differential metabolites and biomarkers also play an important role in the studies. Some researchers indicated that, Maltose in model groups had 498.56 fold change compared with that in control groups [17]. Another study showed levels of 4-hydroxyphenyl acetic acid and hippuric acid is reverse correlation with the decline in renal function by contrast [16]. These conclusions have irreplaceable significance in the study of DN.

The related metabolic pathways was also well observed in the animal model research of DN. Disturbed metabolic pathways, including $\mathrm{NAD}^{+}$metabolism, purine metabolism, oxidative stress, 
Table 6: Biomarkers reported in the context of DN in animal model researches.

\begin{tabular}{|c|c|c|c|c|c|}
\hline Name & Animal & Source & Method & References & Tendency \\
\hline 2-Oxoisocaproic acid & mice & $\mathrm{U}$ & GC-MS & (Li) & $\downarrow$ \\
\hline 5-Hydroxyproline & mice & S & GC-MS & (Li) & $\downarrow$ \\
\hline 9-Octadecanoic acid & Mice & S & GC-MS & (Li) & $\uparrow$ \\
\hline Arginine & mice & S & GC-MS & $(\mathrm{Li})$ & $\downarrow$ \\
\hline Azelate & mice & $\mathrm{U}$ & GC-MS & $(\mathrm{Li})$ & $\downarrow$ \\
\hline Citrate & $\begin{array}{l}\text { mice } \\
\text { /mice } \\
\text { /monkey } \\
\text { /rat } \\
\text { /rat } \\
\text { /rat }\end{array}$ & $\begin{array}{l}\mathrm{S}, \mathrm{U} \\
/ \mathrm{U} \\
/ \mathrm{U} \\
/ \mathrm{U} \\
\mathrm{S} / \mathrm{U} \\
/ \mathrm{U}\end{array}$ & $\begin{array}{l}\text { GC-MS } \\
\text { /NMR } \\
\text { /NMR } \\
\text { /NMR } \\
\text { /NMR } \\
\text { /NMR }\end{array}$ & $\begin{array}{l}\text { (Li) } \\
/ \text { (Stec) } \\
/ \text { (Liu, 2013) } \\
\text { /(Liu, 2015) } \\
\text { /(Zhao, 2012) } \\
/ \text { (Zhao, 2011) }\end{array}$ & $\begin{array}{l}\uparrow \\
/ \downarrow \\
/ \uparrow \\
/ \uparrow \\
/ \downarrow / \uparrow \\
/ \uparrow\end{array}$ \\
\hline$\alpha$-Ketoglutarate & mice & S & GC-MS & (Li) & $\downarrow$ \\
\hline Eicosatetraenoic acid & mice & S & GC-MS & (Li) & $\uparrow$ \\
\hline Erythronate & mice & $\mathrm{U}$ & GC-MS & (Li) & $\downarrow$ \\
\hline Ethanolamine & mice & $\mathrm{U}$ & GC-MS & (Li) & $\downarrow$ \\
\hline Fumarate & mice/mice/monkey & $\mathrm{S} / \mathrm{U} / \mathrm{U}$ & GC-MS/NMR/NMR & (Li)/(Stec)/(Liu, 2013) & $\downarrow / \downarrow / \uparrow$ \\
\hline Gluconate & mice & $\mathrm{U}$ & GC-MS & (Li) & $\downarrow$ \\
\hline Glucuronate & mice & $\mathrm{U}$ & GC-MS & $(\mathrm{Li})$ & $\downarrow$ \\
\hline Glutarate & mice & $\mathrm{U}$ & GC-MS & (Li) & $\uparrow$ \\
\hline Glycerate & mice & $\mathrm{U}$ & GC-MS & (Li) & $\downarrow$ \\
\hline Hexadecanoic acid & mice & S & GC-MS & (Li) & $\downarrow$ \\
\hline Isoleucine & mice/rat/rat/rat & $\mathrm{S} / \mathrm{S} / \mathrm{U} / \mathrm{R}$ & GC-MS/NMR/NMR/NMR & (Li)/(Liu, 2015) & $\uparrow / \uparrow / \uparrow / \downarrow$ \\
\hline Lysine & mice/rat & $\mathrm{S} / \mathrm{R}$ & GC-MS/NMS & (Li)/(Liu, 2015) & $\downarrow$ \\
\hline Malate & mice & $\mathrm{S}, \mathrm{U}$ & GC-MS & (Li) & $\downarrow$ \\
\hline Methionine & mice/rat/rat & $S / R / S$ & GC-MS/NMR/NMR & (Li)/(Liu, 2015)/(Zhao, 2012) & $\downarrow$ \\
\hline Octadecadienoic acid & mice & S & GC-MS & (Li) & $\uparrow$ \\
\hline Octadecanoic acid & mice & $\mathrm{U}$ & GC-MS & (Li) & $\downarrow$ \\
\hline Succinate & $\begin{array}{l}\text { mice } \\
/ \text { rat } \\
\text { /rat }\end{array}$ & $\begin{array}{l}\mathrm{S}, \mathrm{U} \\
/ \mathrm{U} \\
/ \mathrm{U} / \mathrm{R}\end{array}$ & $\begin{array}{l}\text { GC-MS } \\
\text { /NMR } \\
\text { /NMR }\end{array}$ & $\begin{array}{l}\text { (Li) } \\
\text { /(Zhao, 2012) } \\
\text { /(Zhao, 2011) }\end{array}$ & $\begin{array}{l}\downarrow \\
/ \uparrow \\
/ \uparrow / \downarrow\end{array}$ \\
\hline Tetradecanoic acid & mice & S & GC-MS & (Li) & $\downarrow$ \\
\hline Valine & mice/monkey/rat/rat & $\mathrm{S} / \mathrm{S} / \mathrm{R} / \mathrm{S}$ & GC-MS/NMR/NMR/NMR & $\begin{array}{l}\text { (Li) /(Liu,2013)/(Liu, 2015) / } \\
\text { (Zhao, 2012) }\end{array}$ & $\uparrow / \downarrow / \downarrow / \uparrow$ \\
\hline Cis-aconitate & rat, mice & $\mathrm{U}$ & GC-MS,NMR & (Li),(Stec) & $\downarrow$ \\
\hline \multirow[t]{2}{*}{ Acetate } & monkey & $\mathrm{S} / \mathrm{U}$ & NMR & (Liu, 2013) & $\uparrow / \downarrow$ \\
\hline & rat & $\mathrm{U}$ & NMR & (Liu, 2015) & $\uparrow$ \\
\hline Acetate/Betaine & rat & $\mathrm{R}$ & NMR & (Zhao, 2012) & $\downarrow$ \\
\hline Acetoacetate & monkey & S & NMR & (Liu, 2013) & $\uparrow$ \\
\hline \multirow[t]{2}{*}{ Alanine } & monkey & S & NMR & (Liu, 2013) & $\downarrow$ \\
\hline & rat & S,U,R & NMR, GC-MS & $\begin{array}{l}\text { (Liu, 2015)/(Zhao, 2012)/ } \\
\text { (Zhao, 2015)/(Zhao, 2011) }\end{array}$ & $\downarrow$ \\
\hline Allantoin & monkey/rat & $\mathrm{U} / \mathrm{U}, \mathrm{R}$ & NMR/ NMR, GC-MS & $\begin{array}{l}\text { (Liu, 2013)/ (Liu, 2015)/(Zhao, } \\
\text { 2012)/(Zhao, 2015)/(Zhao, } \\
\text { 2011) }\end{array}$ & $\uparrow$ \\
\hline Formate & monkey & $\mathrm{U}$ & NMR & (Liu, 2013) & $\downarrow$ \\
\hline HDL & monkey & S & NMR & (Liu, 2013) & $\downarrow$ \\
\hline Histidine & monkey/rat & $\mathrm{S} / \mathrm{U} / \mathrm{R}$ & NMR & $\begin{array}{l}\text { (Liu, 2013)/(Liu, 2013)/(Liu, } \\
\text { 2015) }\end{array}$ & $\downarrow$ \\
\hline
\end{tabular}




\begin{tabular}{|c|c|c|c|c|c|}
\hline Lipids & monkey/rat & S & NMR & (Liu, 2013)/(Liu, 2015) & $\uparrow$ \\
\hline $\mathrm{NAD}+$ & monkey & $\mathrm{U}$ & NMR & (Liu, 2013) & $\downarrow$ \\
\hline Pyruvate & monkey/rat/rat & $\mathrm{S} / \mathrm{U} / \mathrm{S}$ & NMR & $\begin{array}{l}\text { (Liu, 2013)/(Liu, 2015) } \\
\text { /(Zhao, 2012) }\end{array}$ & $\downarrow$ \\
\hline TMA & monkey/rat & $\mathrm{U}$ & NMR & (Liu, 2013)/(Liu, 2015) & $\uparrow / \downarrow$ \\
\hline Phenylalanine & monkey/rat & $\mathrm{U} / \mathrm{R}$ & NMR & (Liu, 2013)/ (Liu, 2015) & $\uparrow / \downarrow$ \\
\hline Tyrosine & monkey/rat & $S / R$ & NMR & (Liu, 2013)/ (Liu, 2015) & $\downarrow$ \\
\hline Unsaturated lipids & monkey/rat & S & NMR & (Liu, 2013)/ (Liu, 2015) & $\uparrow$ \\
\hline Benzoate & monkey/rat & U & NMR & (Liu, 2013)/(Liu, 2015) & $\uparrow$ \\
\hline Glutamate & monkey/rat & $S / R$ & NMR & $\begin{array}{l}\text { (Liu, 2013)/(Liu, 2015) } \\
\text { / (Zhao, 2012)/ (Zhao, 2011) }\end{array}$ & $\downarrow$ \\
\hline acetone & rat & S & NMR & (Liu, 2015) & $\uparrow$ \\
\hline creatinine /ethanol & rat & $\mathrm{U} / \mathrm{R}$ & NMR & (Liu, 2015) & $\downarrow / \uparrow$ \\
\hline Glutamine/ NAG & rat & S & NMR & (Liu, 2015) & $\downarrow / \uparrow$ \\
\hline VLDL/LDL & rat, monkey & S & NMR & $\begin{array}{l}(\text { Liu, 2015)/(Liu, 2013)/ } \\
\text { (Zhao, 2012) }\end{array}$ & $\uparrow$ \\
\hline choline & rat & S,R & NMR & (Liu, 2015)/(Zhao, 2012) & $\downarrow$ \\
\hline creatine & rat & S,U,R & NMR & $\begin{array}{l}\text { (Liu, 2015)/(Zhao, 2012)/ } \\
\text { (Zhao, 2011) }\end{array}$ & $\downarrow$ \\
\hline 3-HB & rat & $S, R, U$ & NMR,GC-MS & $\begin{array}{l}\text { (Liu, 2015)/(Zhao, 2012)/ } \\
\text { (Zhao, 2011),(Li) }\end{array}$ & $\uparrow$ \\
\hline DMA & rat & $\mathrm{U}$ & NMR & (Liu, 2015)/ (Zhao, 2011) & $\downarrow$ \\
\hline $\begin{array}{l}\text { 2-oxoglutarate/2-oxoisocaproate/3- } \\
\text { indoxyl sulfate/4-hydroxyphenyl } \\
\text { acetate }\end{array}$ & mice & U & NMR & (Stec) & $\downarrow$ \\
\hline Hippurate & $\begin{array}{l}\text { mice } \\
\text { /monkey/rat } \\
\text { /rat }\end{array}$ & $\begin{array}{l}\mathrm{U} \\
/ \mathrm{U} \\
/ \mathrm{R} / \mathrm{U}\end{array}$ & $\begin{array}{l}\text { NMR } \\
\text { /NMR } \\
\text { /GC-MS/LC-MS/NMR }\end{array}$ & $\begin{array}{l}\text { (Stec) } \\
/ \text { (Liu, 2013) /(Zhao, 2012) } \\
/(\text { Zhao, 2015) /(Zhao, 2011) }\end{array}$ & $\begin{array}{l}\downarrow \\
/ \uparrow \\
/ \uparrow\end{array}$ \\
\hline $\mathrm{N}$-phenyl-acetylglycine & mice & $\mathrm{U}$ & NMR & (Stec) & $\downarrow$ \\
\hline GPC & rat & $\mathrm{R}$ & NMR & (Zhao, 2011) & $\downarrow$ \\
\hline 2-ketoglutarate & rat & $\mathrm{U}$ & NMR & (Zhao, 2012) & $\uparrow$ \\
\hline Dimethylamine/LeupIle & rat & $\mathrm{U} / \mathrm{S}$ & NMR & (Zhao, 2012) & $\downarrow / \uparrow$ \\
\hline Glucose & rat & $\mathrm{R}$ & NMR/ GC-MS & (Zhao, 2012)/ (Zhao, 2015) & $\uparrow$ \\
\hline 11,14-Eicosadienoic acid & rat & $\mathrm{R}$ & LC-MS & (Zhao, 2015) & $\uparrow$ \\
\hline $\begin{array}{l}\text { 2,3-Dihydroxybutanoic acid } \\
\text { (4-Deoxythreonic acid) }\end{array}$ & rat & $\mathrm{R}$ & GC-MS & (Zhao, 2015) & $\downarrow$ \\
\hline $\begin{array}{l}\text { 2,8-Dihydroxyquinolinebeta-d- } \\
\text { glucuronide/ } \\
\text { 3-Indole carboxylic acid glucuronide }\end{array}$ & rat & $\mathrm{R}$ & LC-MS & (Zhao, 2015) & $\uparrow$ \\
\hline $\begin{array}{l}\text { 2-Methylbutyroylcarnitine/ } \\
\text { Isovalerylcarnitine/Valerylcarnitine }\end{array}$ & rat & $\mathrm{R}$ & LC-MS & (Zhao, 2015) & $\uparrow$ \\
\hline 2-Phenylethanolglucuronide & rat & $\mathrm{R}$ & LC-MS & (Zhao, 2015) & $\uparrow$ \\
\hline 4-Hydroxybutyric acid & rat & $\mathrm{R}$ & GC-MS & (Zhao, 2015) & $\uparrow$ \\
\hline 4-Hydroxy-L-proline & rat & $\mathrm{R}$ & GC-MS & (Zhao, 2015) & $\downarrow$ \\
\hline 4-Pyridoxic acid & rat & $\mathrm{R}$ & LC-MS & (Zhao, 2015) & $\uparrow$ \\
\hline $\begin{array}{l}\text { 6-Hydroxy-5-methoxyindole } \\
\text { glucuronide/5-Hydroxy-6- } \\
\text { methoxyindole glucuronide }\end{array}$ & rat & $\mathrm{R}$ & LC-MS & (Zhao, 2015) & $\uparrow$ \\
\hline $\begin{array}{l}\text { 8,11,14-Eicosatrienoic acid/5,8,11- } \\
\text { Eicosatrienoic acid }\end{array}$ & rat & $\mathrm{R}$ & LC-MS & (Zhao, 2015) & $\uparrow$ \\
\hline Arachidonic acid & rat & $\mathrm{R}$ & GC-MS & (Zhao, 2015) & $\downarrow$ \\
\hline
\end{tabular}




\begin{tabular}{|c|c|c|c|c|c|}
\hline Cholesterol sulfate & rat & $\mathrm{R}$ & LC-MS & (Zhao, 2015) & $\downarrow$ \\
\hline Cholic acid/other isomers & rat & $\mathrm{R}$ & LC-MS & (Zhao, 2015) & $\uparrow$ \\
\hline Citric acid & rat & $\mathrm{R}$ & GC-MS & (Zhao, 2015) & $\uparrow$ \\
\hline d-Fructose & rat & $\mathrm{R}$ & GC-MS & (Zhao, 2015) & $\uparrow$ \\
\hline $\begin{array}{l}\text { d-Glucose/d-Galactose/d-Mannose/ } \\
\text { d-Fructose/Myoinositol/3- } \\
\text { Deoxyarabinohexonic acid }\end{array}$ & rat & $\mathrm{R}$ & LC-MS & (Zhao, 2015) & $\uparrow$ \\
\hline $\begin{array}{l}\text { Docosapentaenoic acid(22n-6)/ } \\
\text { Clupanodonic acid } \\
/ 4,7,10,13,16 \text {-Docosapentaenoic } \\
\text { acid }\end{array}$ & rat & $\mathrm{R}$ & LC-MS & (Zhao, 2015) & $\uparrow$ \\
\hline d-Pyroglutamic acid & rat & $\mathrm{R}$ & GC-MS & (Zhao, 2015) & $\downarrow$ \\
\hline d-Sorbitol & rat & $\mathrm{R}$ & GC-MS & (Zhao, 2015) & $\uparrow$ \\
\hline Elaidic acid & rat & $\mathrm{R}$ & GC-MS & (Zhao, 2015) & $\downarrow$ \\
\hline Fumaric acid & rat & $\mathrm{R}$ & GC-MS & (Zhao, 2015) & $\uparrow$ \\
\hline Glycocholic acid & rat & $\mathrm{R}$ & LC-MS & (Zhao, 2015) & $\uparrow$ \\
\hline Hypotaurinary & rat & $\mathrm{R}$ & GC-MS & (Zhao, 2015) & $\downarrow$ \\
\hline Indoxyl sulfate & rat & $\mathrm{R}$ & LC-MS & (Zhao, 2015) & $\uparrow$ \\
\hline L-(-)-Arabitol & rat & $\mathrm{R}$ & GC-MS & (Zhao, 2015) & $\uparrow$ \\
\hline $\begin{array}{l}\text { Lactic acid/Hydroxypropionic acid/ } \\
\text { Glyceraldehyde }\end{array}$ & rat & $\mathrm{R}$ & GC-MS/LC-MS & (Zhao, 2015) & $\uparrow$ \\
\hline 1-Alpha-aminobutyric acid & rat & $\mathrm{R}$ & GC-MS & (Zhao, 2015) & $\downarrow$ \\
\hline 1-Aspartic acid & rat & $\mathrm{R}$ & GC-MS & (Zhao, 2015) & $\downarrow$ \\
\hline $\begin{array}{l}\text { Linoelaidyl carnitine/Linoleyl } \\
\text { carnitine }\end{array}$ & rat & $\mathrm{R}$ & LC-MS & (Zhao, 2015) & $\uparrow$ \\
\hline $\begin{array}{l}\text { Linoleicacid/Bovinic } \\
\text { acid/9E,11EOctadecadienoic } \\
\text { acid/10E,12ZOctadecadienoic acid/ } \\
\text { Linoelaidic acid }\end{array}$ & rat & $\mathrm{R}$ & LC-MS & (Zhao, 2015) & $\uparrow$ \\
\hline -Lysine & rat & $\mathrm{R}$ & GC-MS & (Zhao, 2015) & $\downarrow$ \\
\hline 1-Methionine & rat & $\mathrm{R}$ & GC-MS & (Zhao, 2015) & $\downarrow$ \\
\hline 1-Ornithine & rat & $\mathrm{R}$ & GC-MS & (Zhao, 2015) & $\downarrow$ \\
\hline L-Palmitoylcarnitine & rat & $\mathrm{R}$ & LC-MS & (Zhao, 2015) & $\uparrow$ \\
\hline L-Threitol & rat & $\mathrm{R}$ & GC-MS & (Zhao, 2015) & $\uparrow$ \\
\hline LysoPC(16:1) & rat & $\mathrm{R}$ & LC-MS & (Zhao, 2015) & $\downarrow$ \\
\hline LysoPC(20:4) & rat & $\mathrm{R}$ & LC-MS & (Zhao, 2015) & $\downarrow$ \\
\hline LysoPE(18:1) & rat & $\mathrm{R}$ & LC-MS & (Zhao, 2015) & $\downarrow$ \\
\hline LysoPE(20:4) & rat & $\mathrm{R}$ & LC-MS & (Zhao, 2015) & $\downarrow$ \\
\hline LysoPI(16:0) & rat & $\mathrm{R}$ & LC-MS & (Zhao, 2015) & $\downarrow$ \\
\hline Malic acid & rat & $\mathrm{R}$ & GC-MS & (Zhao, 2015) & $\uparrow$ \\
\hline Maltose & rat & $\mathrm{R}$ & GC-MS & (Zhao, 2015) & $\uparrow$ \\
\hline Mannose & rat & $\mathrm{R}$ & GC-MS & (Zhao, 2015) & $\uparrow$ \\
\hline Nicotinamide & rat & $\mathrm{R}$ & GC-MS & (Zhao, 2015) & $\downarrow$ \\
\hline $\begin{array}{l}\text { Oleoylcarnitine/Vaccenyl carnitine/ } \\
\text { Elaidic carnitine } \\
\text { /11ZOctadecenylcarnitine }\end{array}$ & rat & $\mathrm{R}$ & LC-MS & (Zhao, 2015) & $\uparrow$ \\
\hline Oxalic acid & rat & $\mathrm{R}$ & GC-MS & (Zhao, 2015) & $\downarrow$ \\
\hline Phosphate & rat & $\mathrm{R}$ & GC-MS & (Zhao, 2015) & $\downarrow$ \\
\hline Serine & rat & $\mathrm{R}$ & GC-MS & (Zhao, 2015) & $\downarrow$ \\
\hline Succinic acid & rat & $\mathrm{R}$ & GC-MS & (Zhao, 2015) & $\uparrow$ \\
\hline
\end{tabular}




\begin{tabular}{|c|c|c|c|c|c|}
\hline Taurine & rat & $\mathrm{R}$ & LC-MS & (Zhao, 2015) & $\downarrow$ \\
\hline Tetracosahexaenoic acid & rat & $\mathrm{R}$ & LC-MS & (Zhao, 2015) & $\uparrow$ \\
\hline Threonic acid & rat & $\mathrm{R}$ & GC-MS & (Zhao, 2015) & $\uparrow$ \\
\hline Threonine & rat & $\mathrm{R}$ & GC-MS & (Zhao, 2015) & $\downarrow$ \\
\hline Urea & rat & $\mathrm{R}$ & GC-MS & (Zhao, 2015) & $\uparrow$ \\
\hline Uric acid & rat & $\mathrm{R}$ & GC-MS & (Zhao, 2015) & $\uparrow$ \\
\hline N-Acetyl glucosamine & rat & $\mathrm{R} / \mathrm{U}$ & GC-MS/NMR & (Zhao, 2015)/ (Liu,2015) & $\uparrow / \downarrow$ \\
\hline Myo-inositol & rat & $\mathrm{R}$ & GC-MS/NMR & (Zhao, 2015)/ (Zhao, 2011) & $\downarrow$ \\
\hline lactate & rat & $\begin{array}{l}\mathrm{R} / \mathrm{S} / \mathrm{U} / \mathrm{R} / \\
\mathrm{U} / \mathrm{R}\end{array}$ & NMR & $\begin{array}{l}\text { /(Liu, 2015)/(Zhao, 2012)/ } \\
(\text { Zhao, 2012)/(Zhao, 2012)/ } \\
(\text { Zhao, 2011)/ } \\
\text { /(Zhao, 2011) }\end{array}$ & $\begin{array}{l}\uparrow / \downarrow / \uparrow \\
/ \uparrow / \uparrow / \uparrow\end{array}$ \\
\hline
\end{tabular}

P: plasma; U: serum; U: urine; R: renal extracts.

lipid metabolism, and renal tubular reabsorption, were further identified, which provide a view to reveal the metabolic changes in DN [8]. Glycolysis and the subsequent TCA cycle was proved to be of crucial importance to approach the underlying mechanism of DN $[10,16]$. The kidney extracts from DN rats showed a decreased level of succinate (a TCA cycle intermediate), implying the glucose oxidation pathway was inhibited in the kidney tissue [21].

\section{Application}

It is very important for the early diagnosis of DN. A large number of studies have suggested some biomarkers could be used in early diagnosis, for example potential FAs biomarkers (both NEFAs and EFAs) concerned with the development of DM to phases of DN [18]. The level of TCA intermediates (especially for citrate and malate) in the urine relative to that in the serum may be an indicator of kidney function, and a potential measure for the early diagnosis of DN [10]. And urinary hippurate has been reported to be an early biomarker of nephrotoxicity $[11,16]$. In addition, phospholipids, IDL and LDL lipids, and unsaturated fatty acids are increased in the early phase of the disease, but not in the late phase [19]. The combined using of five metabolites including $\gamma$-butyrobetaine, SDMA, azelaic acid, MID 114, and MID 127 has a higher value for diagnosis of DN than single markers [20]. All of these differential metabolites are likely to be used for early diagnosis of DN.

As for the progression of DN, adenosine, inosine, uric acid, xanthine and creatinine may be the potential biomarkers for the diagnosis of progression of DN [23]. Especially creatinine is widely used as a marker of DN that reflects the renal function [20]. UA and allantoin may be used as potential markers for oxidative stress in DN [8]. But enoylcarnitinein plasma, urinary glutamine, histidine, urinary tyrosine and urinary hexose may be related to progressive renal dysfunction [22]. In addition, Sphingomyelin (SM) and phosphatidylinositol (PI) two novel biomarkers can be used as indicators for predicting the progress of diabetic nephropathy [12]. And the disturbed purine metabolism and its related XO pathway were involved in the development of DN [7].

Study on the DN progression and pathogenesis can be well applied in the treatment of DN. Some researchers indicated that decreased $\mathrm{NAD}^{+}$has been related to increased oxidative damage and reduced activity of silent information regulator 1 (Sirt1) in aged humans. $\mathrm{NAD}^{+}$depletion and its associated Sirt1 dysfunction may contribute to the pathogenesis of DN. Thus Sirt1 activation may be a new therapeutic target for preventing the onset and progression of DN [8]. Another example alanine, whether the experiments on rats or monkeys, its level is always lower in DN group $[7,8,11,17,21]$. This suggests that alanine is likely to be a suitable biomarker for the prevention, diagnosis and treatment of DN.

\section{Conclusions}

Metabonomics studies in DN have been started since 2006. These studies provide new methods and insights for the early diagnosis and treatment of DN. Nowadays, a lot of metabolites and biomarkers have been used in clinical detection. This review focused on the role of various metabolites and biomarkers of DN, including amino acid, lipid, carbohydrate and nucleotide.

We obtained a number of differential metabolic markers from a wide variety of samples, and explored the related metabolic pathways. These may be of benefit to the prevention, diagnosis and treatment of DN in clinical.

\section{Recommendations}

More studies should be done in the future. First of all, what is the normal range of these biomarkers? How to diagnose DN using these biomarkers? Targeted metabolomics should be done to test the important and great changing biomarkers. Secondly, metabonomics is just a hint of the whole study in DN. It is also important to explore what has happened inside the body. Furthermore, the number of cases in clinical metabolomics is limited and centralized. Further metabolomics tests with multicenter and larger number samples are necessary. In addition, investigate on the upper link of DN in genomics and proteomics is of great significance.

\section{References}

1. Xie Y, Chen X. Epidemiology, major outcomes, risk factors, prevention and management of chronic kidney disease in China. Am J Nephrol. 2008; 28(1):1-7. 
2. Fiehn 0. Combine genomics, metabolome analysis and biochemical modelling to understand metabolic networks. Comp. Funct. Genom. 2001. 2(3):155-168. //dx.doi.org/10.1002/cfg.82.

3. Lindon J C, Holmes E, Nicholson J K. Metabonomics techniques and applications to pharmaceutical research and development. Pharm Res. 2006; 23(6):1075-1088.

4. Liu H, Wang Z, Chen Y. Metabonomics research methods and its prospect of application in the field of radiation medicine. Chin. J. Radiol. Med. Prot. 2012; 32(2):221-224.

5. Whiting DR, Guariguata L, Weil C, Shaw J. IDF diabetes atlas: global estimates of the prevalence of diabetes for 2011 and 2030. Diabetes Res Clin Pract. 2011; 94(3):311-321. doi: 10.1016/j.diabres.2011.10.029.

6. Wang Y, Wan H, Liu CL. The diagnostic value of different time levels of proteinuria for kidney damage in early diabetic nephropathy. 2014; Lab. Med. Clin. 11:1566-1567.

7. Liu J, Wang C, Liu F, Lu Y, Cheng J. Metabonomics revealed xanthine oxidase-induced oxidative stressand inflammation in the pathogenesis of diabetic nephropathy. Anal Bioanal Chem. 2015; 407(9):25692579. doi: 10.1007/s00216-015-8481-0.

8. Liu J, Wang D, Chen Y, Sun H, He S, Wang C, et al. ${ }^{1} \mathrm{H}$ NMR-based metabonomic analysis of serum and urine in a non human primate model of diabetic nephropathy. Mol Biosyst. 2013; 9(11):2645-2652.

9. Sharma K, Karl B, Mathew AV, Gangoiti JA, Wassel CL, Saito R, et al Metabolomics Reveals Signature of Mitochondrial Dysfunction in Diabetic Kidney Disease. J Am Soc Nephrol. 2013; 24(11):1901-1912. doi: 10.1681/ASN.2013020126.

10. Li M, Wang X, Aa J, Qin W, Zha W, Ge Y, et al. GC/TOFMS analysis of metabolites in serum and urine reveals metabolic perturbation of TCA cycle in $\mathrm{db} / \mathrm{db}$ mice involved in diabetic nephropathy. Am J Physiol Renal Physiol. 2013; 304(11):F1317-1324. doi: 10.1152/ ajprenal.00536.2012.

11. Zhao L, Gao H, Zhao Y, Lin D. Metabonomic analysis of the therapeutic effect of Zhi bai Dihuang Pill in treatment of streptozotocin-induced diabetic nephropathy. J Ethnopharmacol. 2012; 142(3):647-56. doi: 10.1016/j.jep.2012.05.031.

12.Zhu C, Liang Q, Hu P, Wang Y, Luo G. Phospholipidomic identification of potential plasma biomarkers associated with type 2 diabetes mellitus and diabetic nephropathy. Talanta. 2011; 85(4):1711-1720. doi: 10.1016/j.talanta.2011.05.036.

13.Zhang J, Yan L, Chen W, Lin L, Song X, Yan X, et al. Metabonomics research of diabetic nephropathy and type 2 diabetes mellitus based on UPLC-oaTOF-MS system. Anal Chim Acta. 2009; 650(1):16-22. doi: 10.1016/j.aca.2009.02.027.
14. Mäkinen V, Soininen P, Forsblom C, Parkkonen M, Ingman P, Kaski $\mathrm{K}$, et al. ${ }^{1} \mathrm{H}$ NMR metabonomics approach to the disease continuum of diabetic complications and premature death. Mol Syst Biol. 2008; 4:167. doi: $10.1038 / \mathrm{msb} 4100205$.

15. Mäkinen V, Soininen P, Forsblom C, Parkkonen M, Ingman P, Kaski $\mathrm{K}$, et al. Diagnosing diabetic nephropathy by ${ }^{1} \mathrm{H}$ NMR metabonomics of serum. MAGMA. 2006; 19(6):281-296.

16. Stec DF, Wang S, Stothers C, Avance J, Denson D, Harris R, et al. Alterations of urinary metabolite profile in model diabetic nephropathy. Biochem Biophys Res Commun. 2015; 456(2):610-614. doi: 10.1016/j.bbrc.2014.12.003.

17.Zhao $\mathrm{T}$, Zhang $\mathrm{H}$, Zhao $\mathrm{T}$, Zhang $\mathrm{X}, \mathrm{Lu} \mathrm{J}$, Yin $\mathrm{T}$, et al. Intrarenal metabolomics reveals the association of local organic toxins with the progression of diabetic kidney disease. J Pharm Biomed Anal. 2012; 60:32-43. doi: 10.1016/j.jpba.2011.11.010.

18. Han L, Xia J, Liang Q, Wang Y, Wang Y, Hua P, et al. Plasma esterified and non-esterified fatty acids metabolic profiling using gas chromatography-mass spectrometry and its application in the study of diabetic mellitus and diabetic nephropathy. Anal Chim Acta. 2011; 689(1):85-91. doi: 10.1016/j.aca.2011.01.034.

19. MäkinenV, Tynkkynen T, SoininenP, PeltolaT, Kangas AJ, Forsblom C, et al. Metabolic Diversity of Progressive Kidney Disease in 325 Patients with Type 1 Diabetes. J Proteome Res. 2012; 11(3):17821790. doi: $10.1021 /$ pr201036j.

20. Hirayama A, Nakashima E, Sugimoto M, Akiyama S, Sato W, Maruyama $S$, et al. Metabolic profiling reveals new serum biomarkers for differentiating diabetic nephropathy. Anal Bioanal Chem. 2012; 404(10):3101-3109. doi: 10.1007/s00216-012-6412-x.

21. Zhao L, Gao H, Lian F, Liu X, Zhao Y, Lin D. 1H-NMR-based metabonomic analysis of metabolic profiling in diabetic nephropathy rats induced by streptozotocin. Am J Physiol Renal Physiol. 2011; 300(4):F947956. doi: 10.1152/ajprenal.00551.2010.

22. Pena MJ, Heerspink HJL, Hellemons ME, Friedrich T, Dallmann G, Lajer $\mathrm{M}$, et al. Urine and plasma metabolites predict the development of diabetic nephropathy in individuals with Type 2 diabetes mellitus. Diabet Med. 2014; 31(9):1138-1147. doi: 10.1111/dme.12447.

23. Xia J, Liang Q, Liang X, Wang Y, Hu P, Li P, et al. Ultraviolet and tandem mass spectrometry for simultaneous quantification of 21 pivotal metabolites in plasma from patients with diabetic nephropathy. J Chromatogr B Analyt Technol Biomed Life Sci. 2009; 877(2021):1930-1936. doi: 10.1016/j.jchromb.2009.05.047. 